\title{
COPENHAGEN RADIOCARBON MEASUREMENTS VIII GEOGRAPHIC VARIATIONS IN ATMOSPHERIC $\mathrm{C}^{1+}$ ACTIVITY
}

\author{
HENRIK TAUBER
}

Department of Natural Sciences, National Museum, Copenhagen

The following list covers measurements of bomb-produced $\mathrm{C}^{14}$ in series of cereals and grasses collected on the northern hemisphere from 1956 through 1966. Samples were measured in order to determine the yearly addition of bomb produced $\mathrm{C}^{14}$ at a single locality, and to detect possible geographic variations in the distribution and uptake of bombgenerated $\mathrm{C}^{14}$ in terrestrial plant material during years with greatly varying additions of bomb $\mathrm{C}^{14}$. Measurements are given in the $\Delta$ scale (Lamont VIII), i.e. per mil deviations from 0.95 times the activity of the NBS oxalic-acid standard (the natural $\mathrm{C}^{14}$ level).

\section{ACKNOWLEDGMENTS}

The work has been supported by two grants from the Danish State Research Foundation. $\mathrm{C}^{13} / \mathrm{C}^{12}$ ratios of the samples were kindly measured by J. Møller, Physical Laboratory II, H. C. Ørsted Institute, University of Copenhagen. Grass samples collected in 1960 and samples from Greenland, 1963, have been obtained through the courtesy of a large number of institutions, $\mathrm{C}^{14}$ stations, and individuals.

\section{SAMPLE DESCRIPTIONS}

\section{A. Post-bomb rise in $C^{14}$ activity in Denmark}

\section{Danish cereals 1956 to 1966}

Samples of cereals which grew in locations ca. $10 \mathrm{mi} \mathrm{N}$ of Copenhagen (55 $50^{\prime} \mathrm{N}$ Lat; $12^{\circ} 30^{\prime} \mathrm{E}$ Long), Denmark, in area with rather little industry. Collected on dates close to June 1 or July 1 ; in latter case only new ears of cereals were assayed. Results from July 1 each year are plotted in Fig. 1. Comment: measurements on samples from 1956 through 1959 have previously been reported in $\Delta \mathrm{C}^{14}$ scale (Tauber, 1960). Rise in $\mathrm{C}^{14}$ activity is similar to that reported from other stations in middle latitudes (Lamont VIII; Cambridge IV, VI; Saskatchewan III, IV; Yale VII; Münnich and Vogel, 1963; Nydal, 1963; Stockholm V; Uppsala II, VI; UCLA IV, V; Monaco II). In 1958 and 1959 a latitudinal effect in the rise of $\mathrm{C}^{14}$ was suggested (Tauber, 1961). This effect has not been borne out clearly in subsequent years in these latitudes, and the amplitude and extent of such an effect may depend on meteorological factors that vary from year to year. At European longitudes the polar front, thus, had an extreme northern position during the whole summer of 1959, while it oscillated over a much wider latitudinal range in the following years. 


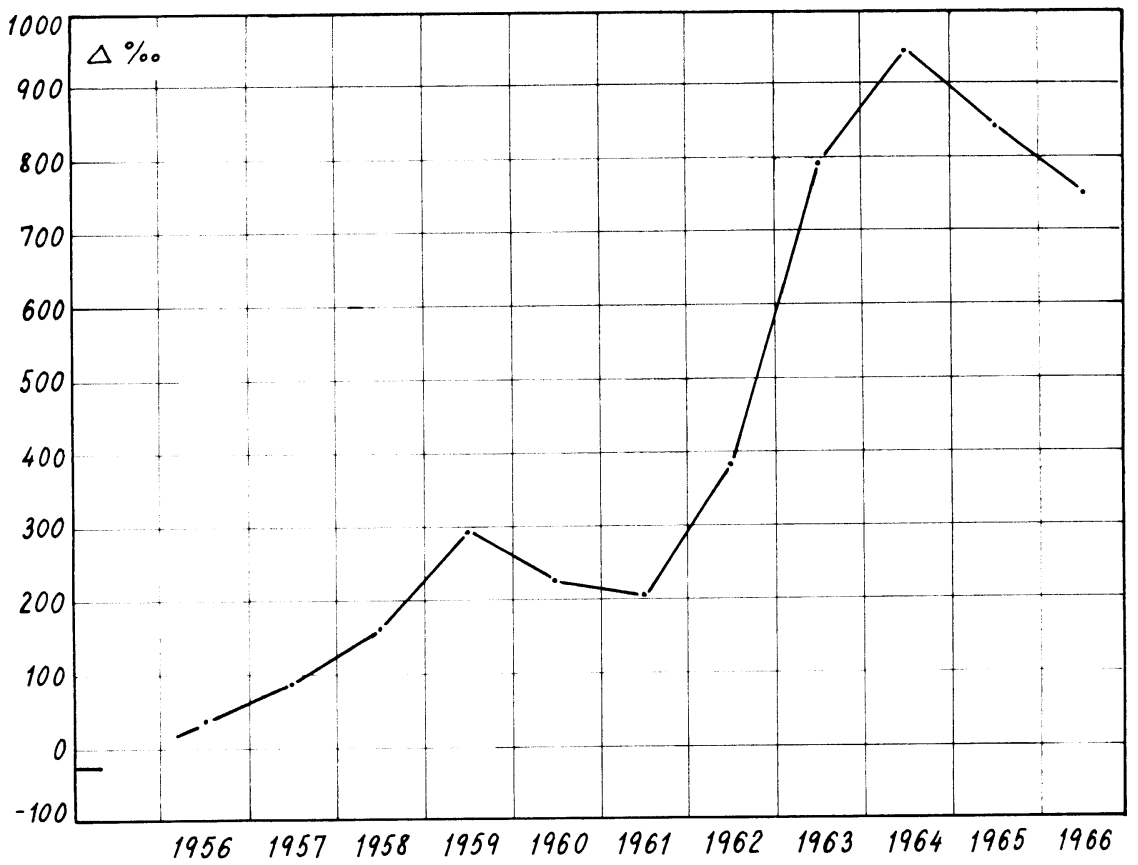

Fig. 1. Per mil $C^{14}$ excess over natural $C^{14}$ level $(\Delta)$ in Danish cereals $(5.5)^{\circ} 50^{\prime}$ $\mathrm{N}$ Lat; $12 \circ 30^{\prime}$ E Long) on July 1 each year from 1956 through 1966 . The activity on July 1 is not the absolute peak value of that year.

$\begin{array}{lccc} & \delta \mathrm{C}^{144} / \% & \delta \mathrm{C}^{13} / \% & \Delta / \% \\ \text { K-612. Frederiksdal 1956 } & \mathbf{3 5} \pm \mathbf{8} & \mathbf{- 2 6 . 2} & \mathbf{3 7} \pm \mathbf{8} \\ \text { Ears of barley, coll. July 1, 1956. } & & & \end{array}$

K-611. Sorgenfri 1957
Ears of barley, coll. July 1, 1957.

$\begin{array}{lllll}\text { K-613. Virum } 1958 & 165 \pm 7 & \mathbf{- 2 4 . 5} & 164 \pm 7\end{array}$

Grains of wheat, exact date of collection not known.

K-610. Frederiksdal 1959

Ears of rye, coll. July 1, 1959.

K-615. Vedbaek 1959

Ears of rye, coll. July 1, 1959.

K-641. Naerum 1960

Ears of oats, coll. July 2, 1960.

K-643. Virum 1960

Ears of barley, coll. July 2, 1960.

$$
82 \pm 7 \quad-26.5 \quad 85=7
$$

$$
292 \pm 7 \quad-25.5 \quad 293 \pm 7
$$$$
293 \pm 9 \quad-26.1 \quad 295 \pm 9
$$$$
222 \pm 8 \quad-25.2 \quad 223 \pm 8
$$$$
233 \pm 8 \quad(-26) \quad 235 \pm 10
$$ 


\section{K-642. Naerum 1961}

$\delta \mathrm{C}^{14 \%} \% \quad \delta \mathrm{C}^{13 \%} \% \quad \Delta \%$

Ears of oats, coll. July 1, 1961.

$$
\mathbf{2 0 5} \pm \mathbf{8} \quad-\mathbf{2 7 . 9} \quad \mathbf{2 1 2} \pm \mathbf{8}
$$

K-644. Naerum 1961

Ears of barley, coll. July 1, 1961.
K-645. Naerum 1962
$336 \pm 8 \quad-27.7$
$\mathbf{3 4 3} \pm \mathbf{8}$

Green parts of oats, coll. June 5, 1962.
K-646. Naerum 1962
$377 \pm 8 \quad-27.9$
$385 \pm 8$

Ears of barley, coll. July 1, 1962.
K-660. Naerum 1963
$666 \pm 9 \quad(-26)$
$669 \pm 11$

Green parts of barley, coll. June 1, 1963.

$\begin{array}{lllll}\text { K-661. Naerum } 1963 & 790 \pm 9 & \mathbf{2 6 . 2} & \mathbf{7 9 4} \pm 9\end{array}$

Ears of barley, coll. July 1, 1963.

K-668. Naerum $1964 \quad 882 \pm 9 \quad(-26) \quad 886 \pm 11$

Green parts of barley, coll. June 1, 1964.

$\begin{array}{lllll}\text { K-667. Naerum } 1964 & 938 \pm 8 & \mathbf{2 6 . 9} & 945 \pm 8\end{array}$

Ears of oats, coll. July 1, 1964.

K-692. Naerum $1965 \quad 794 \pm 9 \quad(-26) \quad 798 \pm 11$

Green parts of barley, coll. June 1, 1965.

$\begin{array}{lllll}\text { K-693. Naerum } 1965 & \mathbf{8 4 0} \pm 9 & (-26) & \mathbf{8 4 4} \pm 11\end{array}$

Ears of barley, coll. July 1, 1965.

K-694. Naerum $1966 \quad 722 \pm 9 \quad(-26) \quad 725 \pm 11$

Green parts of barley, coll. June 1, 1966.

K-695. Naerum $1966 \quad 748 \pm 9 \quad(-26) \quad 752 \pm 11$

Ears of barley, coll. June 28, 1966.

\section{B. Grass samples 1960}

\section{Grass samples from $40^{\circ} \mathrm{N}$ to $80^{\circ} \mathrm{N}$}

Samples of green parts of grasses and cereals collected between July 2 and Aug. 1, 1960, on both sides of Atlantic Ocean in latitudes from $40^{\circ} \mathrm{N}$ to $80^{\circ} \mathrm{N}$. Samples in this and next series were collected in order to trace a possible geographic variation in atmospheric $\mathrm{C}^{14}$ concentration in Northern Hemisphere in a year with a minimal addition of bombgenerated $\mathrm{C}^{14}$ (see Fig. 1). Comment: atmospheric $\mathrm{C}^{11}$ concentrations proved to be remarkably uniform within these latitudes in 1960. Average value for all samples in this series is $\Delta=247 \%$. 
$\delta \mathrm{C}^{14 \%} \% \quad \delta \mathrm{C}^{13 \%} \% 0 \quad \Delta \%$

K-648. Svalbard 78 N $\quad 234 \pm 6 \quad-30.5 \quad 248 \pm 6$

Grass from Cape Linné $\left(78^{\circ} 04^{\prime} \mathrm{N}\right.$ Lat, $13^{\circ} 38^{\prime} \mathrm{E}$ Long), Svalbard. Coll. July 23, 1960.

K-649. Finnmarken $70 \circ \mathbf{N} \quad \mathbf{2 5 0} \pm \mathbf{8} \quad(\mathbf{- 2 5}) \quad \mathbf{2 5 0} \pm \mathbf{1 0}$

Grass from Nesseby $\left(70^{\circ} 09^{\prime} \mathrm{N}\right.$ Lat, $28^{\circ} 55^{\prime}$ E Long), Finnmarken, Norway. Coll. July 4, 1960.

K-650. Trondheim $63{ }^{\circ} \quad 241 \pm 8 \quad(-25) \quad 241 \pm 10$

Ears of wheat from $\mathrm{N}$ of Trondheim $\left(63^{\circ} 24^{\prime} \mathrm{N}\right.$ Lat, $10^{\circ} 22^{\prime} \mathrm{E}$ Long), Norwey. Coll. July 6, 1960.

K-651. Naerum $56^{\circ} \mathrm{N} \quad 237 \pm 6 \quad-25.6 \quad 238 \pm 6$

Grass from Naerum (55 $50^{\prime} \mathrm{N}$ Lat, $12^{\circ} 30^{\prime} \mathrm{E}$ Long), Denmark. Cioll. July 22, 1960.

K-652. Bern $47^{\circ} \mathrm{N} \quad 252 \pm 8 \quad(-25) \quad 252 \pm 10$

Ears of wheat from Grauholz ( $46^{\circ} 56^{\prime} \mathrm{N}$ Lat, $7^{\circ} 26^{\prime} \mathrm{E}$ Long), Bern, Switzerland. Coll. July 16, 1960.

K-653. Abruzzi $42{ }^{\circ} \quad 250 \pm 8 \quad(-25) \quad 250 \pm 10$

Ears of wheat from $1040 \mathrm{~m}$ above sealevel at Castel di Sangro (41 $48^{\prime} \mathrm{N}$ Lat, 14 02' E Long), Abruzzi, Italy. Coll. July 7, 1960.

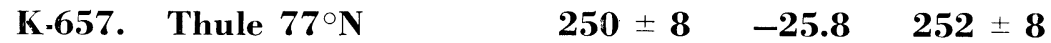

Grass from $10 \mathrm{~km}$ E of Thule Air Base $\left(76^{\circ} 32^{\prime} \mathrm{N} \mathrm{Lat}, 68^{\circ} 40^{\prime} \mathrm{W}\right.$ Long), West Greenland. Coll. Aug. 1, 1960.
K-658. Itivnera $64^{\circ} \mathrm{N}$
$235 \pm 9 \quad-26.9 \quad 240 \pm 9$

Grass from Itivnera (64 $23^{\prime} \mathrm{N}$ Lat, $50^{\circ} 23^{\prime} \mathrm{W}$ Long), West Greenland. Coll. July 2, 1960.

\section{K-659. Churchill $59^{\circ} \mathrm{N} \quad 241 \pm 9 \quad-29.1 \quad 251 \pm 9$}

Grass from Churchill (58 $40^{\prime} \mathrm{N}$ Lat, $94^{\circ} 10^{\prime} \mathrm{W}$ Long), Manitoba, Canada. Coll. July 18, 1960.

\section{Grass samples from $0^{\circ}$ to $40^{\circ} \mathrm{N}$}

Samples of green parts of grasses, and a sample of banana lcaves, collected between July 3 and Aug. 17, 1960, in latitudes from $0^{\circ}$ to $40^{\circ} \mathrm{N}$, for comparison with samples from more northern latitudes. Comment: atmospheric $\mathrm{C}^{14}$ concentrations scattered considerably more in these latitudes than between $40^{\circ} \mathrm{N}$ and $80^{\circ} \mathrm{N}$. Activities appear to some extent to be lower in locations with a dominance of winds from $\mathrm{S}$, and higher in locations with wind components from N. Average value for all samples in series is $\Delta=219 \%$. This is significantly lower than for previous series. Decrease may partly have been caused by advection of air from South- 
ern Hemisphere; a latitudinal effect in transfer of bomb-produced $\mathrm{C}^{14}$ from middle latitudes could therefore only be small in 1960 . The $\delta \mathrm{C}^{13}$ values, except for one sample (K-675), are all outside normal range of terrestrial plant material $(-20$ to $-30 \%$ ). These anomalous values might have been caused by a recycling of $\mathrm{CO}_{2}$ between ocean and atmosphere at these latitudes, or by a release of $\mathrm{CO}_{2}$ from soil carbonates. However, in that case the $\delta \mathrm{C}^{14}$ values should have been more suppressed, since only a small fraction of bomb-produced $\mathrm{C}^{14}$ had entered into ocean surface water in 1960 (Broecker and Olson, 1960), or could have been included in soil carbonates. The unusual isotopic fractionation is rather due to changes in ratio of assimilation of carbon to respiration in the plants, caused by the extreme microclimatic conditions that prevail directly above the soil in warm and dry areas. The only sample in this series that has normal $\mathrm{C}^{13} /{ }^{12}$ ratio, thus, is banana leaves, which will not have been exposed to these microclimatic extremes. A temperature dependence of the $\mathrm{C}^{13} / \mathrm{C}^{12}$ ratios in marine plankton has previously been noted by Sackett et al. (1965).

$$
\text { K-654. Kaduna } 11^{\circ} \mathrm{N} \quad 255 \pm 8 \quad-13.7 \quad 225 \pm 8
$$

Grass from Kaduna $\left(10^{\circ} 34^{\prime} \mathrm{N}\right.$ Lat, $7^{\circ} 22^{\prime} \mathrm{E}$ Long), Nigeria. Coll. July 14, 1960.

$$
\text { K-655. Enugu } 6^{\circ} \mathbf{N} \quad 242 \pm 8 \quad-11.4 \quad 207 \pm 8
$$

Grass from Enugu (6॰ $28^{\prime}$ N Lat, $7^{\circ} 24^{\prime}$ E Long), Nigeria. Coll. July $23,1960$.

$$
\text { K-684. Nora-Pula } 39^{\circ} \mathrm{N} \quad 232 \pm 9 \quad-15.0 \quad 207 \pm 8
$$

Grass from Nora-Pula (39॰ $01^{\prime} \mathrm{N}$ Lat, $9^{\circ} 01^{\prime} \mathrm{E}$ Long), Sardinia, Italy. Coll. July 24, 1960.

$$
\text { K-656. Wad Medani } 14{ }^{\circ} \quad 251 \pm 8 \quad-13.0 \quad 221 \pm 8
$$

Grass from Wad Medani (14 24' $\times$ Lat, $33^{\circ} 30^{\prime}$ E Long), Sudan. Coll. July 8, 1960.

K-677. Karachi $25^{\circ} \mathrm{N} \quad 220 \pm 8 \quad-13.9 \quad 193 \pm 8$

Grass from Zoological Garden in Karachi $\left(24^{\circ} 50^{\prime} \mathrm{N}\right.$ Lat, $67^{\circ} 13^{\prime} \mathrm{E}$ Long), Pakistan. Coll. Aug. 17, 1960.

K-678. Chinsura $23{ }^{\circ} \quad 235 \pm 8 \quad-19.1 \quad 220 \pm 8$

Grass from Chinsura $\left(22^{\circ} 55^{\prime} \mathrm{N}\right.$ Lat, $\left.88^{\circ} 24^{\prime} \mathrm{E} \mathrm{Long}\right), 40 \mathrm{~km} \mathrm{~N}$ of Calcutta, India. Coll. July 10, 1960.

K-679. Ilan Hsien $25^{\circ} \mathrm{N} \quad 260 \pm 9 \quad-11.4 \quad 226 \pm 9$

Maize from coastal field at Ilan Hsien outside of Taipei $\left(25^{\circ} 02^{\prime} \mathrm{N}\right.$ Lat, $121^{\circ} 26^{\prime}$ E Long), Taiwan. Coll. July 14, 1960. 


\section{$\delta \mathrm{C}^{14 \%} \% \quad \delta \mathrm{C}^{13} \%$ \\ $\Delta \% o$ \\ K-680. Seoul $38^{\circ} \mathrm{N}$ \\ $258 \pm 9 \quad-13.9 \quad 230 \pm 9$

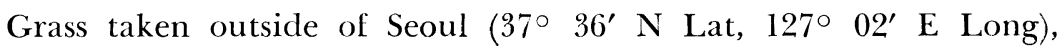
Korea. Coll. Aug. 1, 1960.

K-681. Honolulu $21^{\circ} \quad 270 \pm 8 \quad-11.3 \quad 236 \pm 8$

Grass taken $500 \mathrm{~m}$ above sealevel at Forest Ridge Way outside of Honolulu (21 $20^{\prime} \mathrm{N}$ Lat, $157^{\circ} 51^{\prime} \mathrm{W}$ Long), Hawaii. Coll. July 24, 1960.

K-682. Mexico City $19 \times \mathbf{N} \quad 236 \pm 9 \quad-14.4 \quad 210 \pm 9$

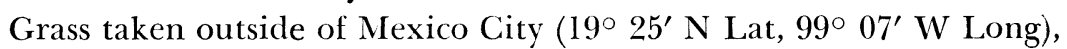
Mexico. Coll. Aug. 2, 1960.

K-683. Coral Gables $26^{\circ} \mathbf{N} \quad 234 \pm 9 \quad-11.4 \quad 200 \pm 9$

Grass from Coral Gables (25 $44^{\prime} \mathrm{N}$ Lat, $80^{\circ} 16^{\prime} \mathrm{W}$ Long), Florida, U.S.A. Coll. July 3, 1960.

\section{K-676. Panama $9{ }^{\circ} \quad 245 \pm 9 \quad-17.1 \quad 225 \pm 9$}

Grass taken at Pacific side of Canal Zone $\left(9^{\circ} 0^{\prime} \mathrm{N}\right.$ Lat, $79^{\circ} 25^{\prime} \mathrm{WW}$ Long), Panama. Coll. July 15, 1960.

\section{K-675. Las Palmas $28{ }^{\circ} \quad 242 \pm 8 \quad-24.6 \quad 241 \pm 8$}

Banana leaves from plantation outside of Las Palmas $\left(28^{\circ} 07^{\prime} \mathrm{N}\right.$ Lat, $15^{\circ} 32^{\prime}$ W Long), Gran Canaria, Canary Islands. Coll. July 22, 1960.

\section{Grass samples 1961}

\section{European series $37^{\circ} \mathrm{N}$ to $70^{\circ} \mathrm{N}$}

Samples of green parts of grasses collected from June 1 through June 13, 1961, on every second degree of latitude between $37^{\circ} \mathrm{N}$ and $70^{\circ} \mathrm{N}$. Samples are assumed to reflect the average $\mathrm{C}^{14}$ activity of the ground level troposphere a few weeks prior to sampling. Series approximates a synchronous profile through Europe in a year with a minimal addition of bomb-produced $\mathrm{C}^{11}$. Samples were taken away from areas with heavy industry, away from main roads, and outside towns and villages; they are named after nearest locality. Comment: apart from a small local Suess effect over Central Europe $\left(44^{\circ} \mathrm{N}\right.$ to $\left.54^{\circ} \mathrm{N}\right)$, $\mathrm{C}^{14}$ activities scatter within statistics. Geographic variations within this sector thus were less than $1 \%$ in 1961. Series of grass samples from 1960 and 1961 suggest that geographic variations in atmospheric $\mathrm{C}^{14}$ in the prebomb state were also below $1 \%$.

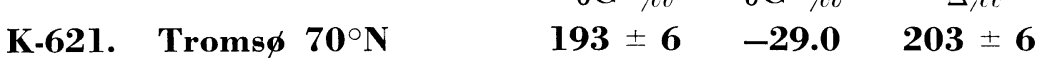

$$
\begin{aligned}
& \text { Grass taken } 2 \mathrm{~km} \mathrm{~S} \text { of Troms } \phi\left(69^{\circ} 38^{\prime} \mathrm{N} \text { Lat, } 19^{\circ} 0^{\prime} \mathrm{E}\right. \text { Long), }
\end{aligned}
$$




\section{$\begin{array}{llccc} & & \delta C^{1} / \% & \delta C^{13} / \% 0 & \Delta \% \\ \text { K-622. Innhavet } 68 \times N & \mathbf{2 0 3} \pm \mathbf{6} & \mathbf{- 2 7 . 5} & \mathbf{2 0 9} \pm \mathbf{6}\end{array}$}

Grass from Innhavet $\left(68^{\circ} 0^{\prime} \mathrm{N}\right.$ Lat, $16^{\circ} 0 \mathrm{l}^{\prime} \mathrm{E}$ Long), Norway. Coll. June 7, 1961.

$$
\text { K-623. Mosjöen } 66^{\circ} \mathrm{N} \quad 202 \pm 6 \quad-26.9 \quad 207 \pm 6
$$

Grass taken $20 \mathrm{~km} \mathrm{~N}$ of Mosjöen (66 $0^{\circ} \mathrm{N}$ Lat, $13^{\circ} 29^{\prime} \mathrm{E}$ Long), Norway. Coll. June 6, 1961.

K-624. Steinkjer $64 . N \quad 198 \pm 8 \quad(-27) \quad 203 \pm 10$

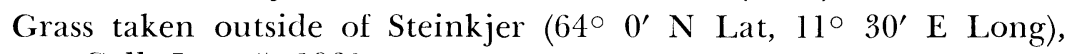
Norway. Coll. June 5, 1961.

\section{K-625. Dovre $62^{\circ} \mathrm{N} \quad 194 \pm 8 \quad(-27) \quad 199 \pm 10$}

Grass from Dovre $\left(62^{\circ} 0^{\prime} \mathrm{N}\right.$ Lat, $9^{\circ} 15^{\prime} \mathrm{E}$ Long), Norway. Coll. June 5, 1961.

K-626. Lilleström $60^{\circ} \quad 192 \pm 8 \quad(-27) \quad 197 \pm 10$

Grass from Lilleström $\left(60^{\circ} 0^{\prime} \mathrm{N}\right.$ Lat, $11^{\circ} 02^{\prime} \mathrm{E}$ Long), Norway. Coll. June 4, 1961.

\section{K-627. Jörlanda 58 N $\quad 209 \pm 6 \quad-28.0 \quad 216 \pm 8$}

Grass from Jörlanda (58 $0^{\prime}$ Lat, $11^{\circ} 50^{\prime}$ E Long), Sweden. Coll. June 3, 1961.

K-628. Kagerup $56^{\circ} \mathrm{N} \quad 205 \pm 6 \quad-28.0 \quad 212 \pm 8$

Grass from Kagerup $\left(56^{\circ} 0^{\prime} \mathrm{N}\right.$ Lat, $12^{\circ} 17^{\prime}$ E Long), Denmark. Coll. June 1, 1961.

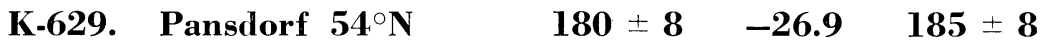

Grass from Pansdorf (54 $0^{\prime} \mathrm{N}$ Lat, $10^{\circ} 43^{\prime}$ E Long), Germany. Coll. June 3, 1961.

K-630. Bad Salzgitter $52{ }^{\circ} \mathbf{N} \quad 188 \pm 8 \quad(-27) \quad 193 \pm 10$

Grass taken $5 \mathrm{~km} \mathrm{SW}$ of Bad Salzgitter $\left(52^{\circ} 0^{\prime} \mathrm{N}\right.$ Lat, $10^{\circ} 14^{\prime} \mathrm{E}$ Long), Germany. Coll. June 4, 1961.

K-631. Karlstadt $50 \circ \mathrm{N} \quad 197 \pm 8 \quad(-27) \quad 202 \pm 10$

Grass taken $6 \mathrm{~km} \mathrm{NE}$ of Karlstadt $\left(50^{\circ} 0^{\prime} \mathrm{N}\right.$ Lat, $9^{\circ} 50^{\prime} \mathrm{E}$ Long), Germany. Coll. June 5, 1961.

K-632. Starnberg $48^{\circ} N \quad 189 \pm 8 \quad(-27) \quad 194 \pm 10$

Grass from Starnberg (48 $0^{\prime} \mathrm{N}$ Lat, $11^{\circ} 21^{\prime} \mathrm{E}$ Long), Germany. Coll. June 6, 1961.

\section{K-633. Primolano $46^{\circ} \mathrm{N} \quad 192 \pm 8 \quad-28.4 \quad 200 \pm 8$}

Grass taken $5 \mathrm{~km} \mathrm{NW}$ of Primolano $\left(46^{\circ} 0^{\prime} \mathrm{N}\right.$ Lat, $11^{\circ} 52^{\prime} \mathrm{E}$ Long), Italy. Coll. June 7, 1961. 


\section{K-634. Riccione $44{ }^{\circ} N \quad 190 \pm 6 \quad-26.8 \quad 199 \pm 6$} June 10.

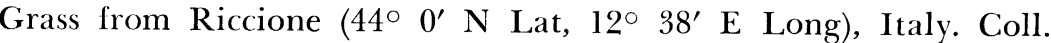

K-635. S. Salvo $42^{\circ} N \quad 231 \pm 6 \quad-17.0 \quad 212 \pm 6$

Grass taken $7 \mathrm{~km}$ SE of S. Salvo $\left(42^{\circ} 0^{\prime} \mathrm{N}\right.$ Lat, $14^{\circ} 46^{\prime} \mathrm{E}$ Long), Italy. Coll. June 10, 1961.

K-636. Sapri $40^{\circ} N \quad 219 \pm 6 \quad-25.7 \quad 221 \pm 6$

Grass taken $12 \mathrm{~km} \mathrm{SE}$ of Sapri $\left(40^{\circ} 0^{\prime} \mathrm{N}\right.$ Lat; $15^{\circ} 42^{\prime} \mathrm{E}$ Long), Italy. Coll. June 11, 1961.

K-637. Ali Marina $38^{\circ} \mathrm{N} \quad 200 \pm 6 \quad-26.7 \quad 204 \pm 6$

Grass from Ali Marina ( $38^{\circ} 0^{\prime} \mathrm{N}$ Lat, $15^{\circ} 22^{\prime} \mathrm{E}$ Long), Sicily, Italy. Coll. June 12, 1961.

K-638. Pachino $37^{\circ} \mathrm{N} \quad 233 \pm 6 \quad-13.2 \quad 204 \pm 6$

Leaves of maize taken $3 \mathrm{~km} \mathrm{~S}$ of Pachino $\left(36^{\circ} 42^{\prime} \mathrm{N}\right.$ Lat, $15^{\circ} 09^{\prime} \mathrm{E}$ Long), Sicily, Italy. Coll. June 13, 1961.

\section{Scandinavian series June 1963}

\section{Grass samples 1963}

Samples of green parts of grasses collected from June 12 through June 17,1963 , on every second degree of latitude between $56^{\circ} \mathrm{N}$ and $70^{\circ} \mathrm{N}$, in Scandinavia. Collected in order to detect possible geographic variations in atmospheric $\mathrm{C}^{14}$ activity in a year with a maximum addition of bomb-produced $\mathrm{C}^{14}$ (see Fig. 1.). Samples were taken with same precautions as in 1961 series. Comment: $\mathrm{C}^{14}$ activities scattered only slightly more than a normal statistical distribution. Even with an extreme addition of bomb-generated $\mathrm{C}^{14}$ a latitudinal effect has thus not been ascertained in this section (compare however Greenlandic series from 1963). Average activity of all samples in series is $\Delta=699 \%$ (compare K-660 and K-661, taken June 1 and July 1, 1963, respectively, in series of Danish cereals 1956 to 1966).

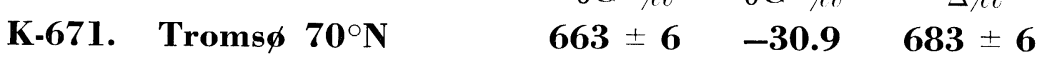

Grass taken $2 \mathrm{~km} \mathrm{~S}$ of Troms $\phi\left(69^{\circ} 38^{\prime} \mathrm{N}\right.$ Lat, $19^{\circ} 0^{\prime} \mathrm{E}$ Long), Norway. Coll. June 17, 1963.

\section{K-670. Innhavet $68^{\circ} \mathrm{N} \quad 681 \pm 6 \quad-29.2 \quad 695 \pm 6$}

Grass from Innhavet $\left(68^{\circ} 0^{\prime} \mathrm{N}\right.$ Lat, $16^{\circ} 01^{\prime} \mathrm{E}$ Long), Norway. Coll. June 16, 1963.

\section{K-669. Mosjöen $66^{\circ} \mathrm{N} \quad 686 \pm 8 \quad-28.7 \quad 698 \pm 8$}

Grass taken $20 \mathrm{~km} \mathrm{~N}$ of Mosjöen (66 $0^{\circ} 0^{\prime} \mathrm{N}$ Lat, $13^{\circ} 29^{\prime} \mathrm{E}$ Long), Norway. Coll. June 15, 1963. 


$$
\begin{aligned}
& \delta \mathrm{C}^{14 \%} \% \quad \delta \mathrm{C}^{13 \%} \% \quad \quad \Delta \% \\
& \text { K-666. Steinkjer 64 } \quad \text { N } \quad 684 \pm 8 \quad-27.5 \quad 692 \pm 8
\end{aligned}
$$

Grass taken outside of Steinkjer $\left(64^{\circ} 0^{\prime} \mathrm{N}\right.$ Lat, $11^{\circ} 30^{\prime} \mathrm{E}$ Long), Norway. Coll. June 15, 1963.

K-665. Dovre $62^{\circ} \mathrm{N} \quad \mathbf{7 0 4} \pm \mathbf{8} \quad-27.5 \quad 713 \pm 8$

Grass from Dovre (62॰ $0^{\prime} \mathrm{N}$ Lat, $9^{\circ} 15^{\prime}$ E Long), Norway. Coll. June 14, 1963.

K-664. Lilleström $60^{\circ} \mathrm{N} \quad \mathbf{7 1 1} \pm \mathbf{8} \quad \mathbf{- 2 8 . 7} \quad \mathbf{7 2 4} \pm \mathbf{8}$

Grass from Lilleström $\left(60^{\circ} 0^{\prime} \mathrm{N}\right.$ Lat, $11^{\circ} 02^{\prime}$ E Long), Norway. Coll. June 13, 1963.

K-663. Jörlanda $588^{\circ} \quad 683 \pm 8 \quad-28.8 \quad 696 \pm 8$

Grass from Jörlanda $\left(58^{\circ} 0^{\prime} \mathrm{N}\right.$ Lat, $11^{\circ} 50^{\prime}$ E Long), Sweden. Coll. June 12, 1963.

K-662. Kvistgaard $56^{\circ} \mathrm{N} \quad 685 \pm 8 \quad-26.7 \quad 691 \pm 8$

Grass from Kvistgaard (56 $0^{\prime} \mathrm{N}$ Lat, $12^{\circ} 30^{\prime} \mathrm{E}$ Long), Denmark. Coll. June 12, 1963.

\section{Greenlandic series June 1963}

Samples of green parts of grasses collected on June 21 and June 26, 1963 , at latitudes of $64^{\circ} \mathrm{N}$ and $82^{\circ} \mathrm{N}$ in Greenland. Collected for comparison with Scandinavian series from same month. Comment: $\mathrm{C}^{14}$ activities were considerably lower than in Scandinavian series, although samples were taken later in month. Simultaneously there was marked decrease in activity from $64^{\circ} \mathrm{N}$ to $82^{\circ} \mathrm{N}$ in Greenland. Compared to Scandinavian series a geographic variation in $\mathrm{C}^{14}$ activity exceeding $14 \%$ of natural $\mathrm{C}^{14}$ level was present in high latitudes of Northern Hemisphere in 1963. Activities of K-686 and K-687 are far below activity at Copenhagen on June 1 (K-660). Delay thus is more than 1 month. Similar delay between northern Sweden and Svalbard in 1963 has previously been reported (Uppsala VI).

$$
\delta \mathrm{C}^{1+4} / \% 0 \quad \delta \mathrm{C}^{13 \%} / \% \quad \Delta \%
$$

K-686. Bronlund Fjord $82 \circ \mathrm{N} \quad \mathbf{5 5 5} \pm \mathbf{6} \quad \mathbf{- 2 6 . 5} \quad \mathbf{5 6 0} \pm \mathbf{6}$

Grass from J $\phi$ rgen Br $\phi$ nlund Fjord $\left(82^{\circ} 10^{\prime} \mathrm{N}\right.$ Lat, $30^{\circ} 30^{\prime} \mathrm{W}$ Long), Peary Land, North Greenland. Coll. June 26, 1963.

\section{K-687. Kapisigdlit $64{ }^{\circ} \quad 605 \pm 6 \quad-28.9 \quad 618 \pm 6$}

Grass taken at mouth of river Kapisigdlit $\left(64^{\circ} 26^{\prime} \mathrm{N}\right.$ Lat, $50^{\circ} 12^{\prime}$ W Long), West Greenland. Coll. June 21, 1963.

\section{Greenlandic series July 1963}

Samples of green parts of grasses collected between July 5 and July 10,1963 , at latitudes from $61^{\circ} \mathrm{N}$ to $73^{\circ} \mathrm{N}$ in Greenland, for comparison 
with previous series from 1963. Comment: activities of Greenlandic samples are intermediate between $\mathrm{C}^{14}$ activities found at Copenhagen on June 1 and July 1, 1963 (compare K-660 and K-661 in series of Danish cereals 1956 to 1966). There is clear gradient in $\mathrm{C}^{14}$ activity from $\mathrm{S}$ to $\mathrm{N}$. At European longitudes preferred position of the polar front in summers is over southern Scandinavia with frequent shifts towards $\mathrm{N}$ and S, while over the Atlantic the polar front is usually found far $S$ of Greenland. Geographic variations in $\mathrm{C}^{14}$ activity found in 1963 are thus consistent with assumption that main transfer of $\mathrm{C}^{14}$ from stratosphere to troposphere takes place at tropopause gaps in middle latitudes, with a subsequent diffusion towards northern and southern latitudes.
K-685. Mestersvig $72 \circ \mathrm{N}$
$\delta \mathrm{C}^{140} \% 0 \quad \delta \mathrm{C}^{13 \%} \% \quad \quad \quad \% \%$
$716 \pm 8 \quad-26.5 \quad 721 \pm 8$
$23^{\circ}$ Grass taken $1 \mathrm{~km}$ away from airport at Mestersvig $\left(72^{\circ} 13^{\prime} \mathrm{N}\right.$ Lat, $3^{\circ} 54^{\prime}$ W Long), East Greenland. Coll. July 19, 1963.

\section{K-688. Kulusuk $65^{\circ} \mathrm{N} \quad \mathbf{7 2 4}^{\circ} \pm \mathbf{8} \quad-28.7 \quad 737 \pm 8$}

Grass from Kulusuk (65 $33^{\prime}$ N Lat, $37^{\circ} 13^{\prime}$ W Long), East Greenland. Coll. July 5, 1963.

\section{K-689. Narssaq $61^{\circ} \mathrm{N} \quad \mathbf{7 6 5} \pm \mathbf{8} \quad \mathbf{- 2 8 . 0} \quad \mathbf{7 7 6} \pm \mathbf{8}$}

Grass from Narssaq $\left(60^{\circ} 58^{\prime} \mathrm{N}\right.$ Lat, $46^{\circ} 05^{\prime} \mathrm{W}$ Long), West Greenland. Coll. July 9, 1963.

Date lists:

Cambridge IV
Cambridge VI
Lamont VIII
Monaco II
Saskatchewan III
Saskatchewan IV
Stockholm V
UCLA IV
UCLA V
Uppsala II
Uppsala VI
Yale VII

\section{REFERENCES}

\author{
Godwin and Willis, 1961 \\ Godwin and Willis, 1964 \\ Broecker and Olson, 1961 \\ Thommeret and Thommeret, 1966 \\ McCallum and Wittenberg, 1962 \\ McCallum and Wittenberg, 1965 \\ Ostlund and Engstrand, 1963 \\ Berger et al., 1965 \\ Berger and Libby, 1966 \\ Olsson, 1960 \\ Olsson and Karlén, 1965 \\ Stuiver and Deevey, 1962
}

Berger, R., Ferguson, G. J., and Libby, W. F., 1965, UCLA radiocarbon dates IV: Radiocarbon, v. 7, p. 336-371. Berger, R., and Libby, W. F., 1966, UCLA radiocarbon dates V: Radiocarbon, v. 8,
p. 467-497.

Broecker, W. S., and Olson, E. A., 1960, Radiocarbon from nuclear tests II: Science, v. 132, p. 712-721.

1961, Lamont radiocarbon measurements VIII: Radiocarbon, v. 3, p. 176-204.

Godwin, H., and Willis, E. H., 1961, Cambridge University natural radiocarbon measurements IV, nuclear-weapon testing and the atmospheric radiocarbon concentration: Radiocarbon, v. 3, p. 77-80. 1964, Cambridge University natural radiocarbon measurements VI: Radio-
carbon, v. 6, p. 116-137.

McCallum, K. J., and Wittenberg, J., 1962, University of Saskatchewan radiocarbon dates III: Radiocarbon, v. 4, p. 71-80. 
1965, University of Saskatchewan radiocarbon dates IV: Radiocarbon, v. 7, p. 229-235.

Münnich, K. O., and Vogel, J. C., 1963, Investigations of meridional transport in the troposphere by means of carbon-14 measurements: Radioactive dating, I.A.E.A., Vienna 1963, p. 189-197.

Nydal, R., 1963, Increase in radiocarbon from the most recent series of thermonuclear tests: Nature, v. 200, no. 4903, p. 212-214.

Olsson, I., 1960, Uppsala natural radiocarbon measurements II: Am. Jour. Sci. Radioc. Supp., v. 2, p. 112-128.

Olsson, I. U., and Karlén, I., 1965, Uppsala radiocarbon measurements VI: Radiocarbon, v. 7, p. 331-335.

Östlund, H. G., and Engstrand, L. G., 1963, Stockholm natural radiocarbon measurements V: Radiocarbon, v. 5, p. 203-227.

Sackett, W. M., Eckelmann, W. R., Bender, M. L., and Bé, A.W.H., 1965, Temperature dependence of carbon isotope composition in marine plankton and sediments: Science, v. 148, p. 235-237.

Stuiver, M., and Deevey, E. S., 1962, Yale natural radiocarbon measurements VII: Radiocarbon, v. 4, p. 250-262.

Tauber, H., 1960, Post-bomb rise in radiocarbon activity in Denmark: Science, v. 131, p. 921-922.

1961, Latitudinal effect in the transfer of radiocarbon from stratosphere to troposphere: Science, v. 133, p. 461-462.

Thommeret, J., and Thommeret, Y., 1966 Monaco radiocarbon measurements II: Radiocarbon, v. 8, p. 286-291. 